\title{
Impedimetric Characterization of Bipolar Nanoelectrodes with Cancer Cells
}

\author{
Andie J. Robinson, Akhil Jain, Ruman Rahman, Sidahmed Abayzeed,* Richard J. M. Hague, \\ and Frankie J. Rawson*
}

Cite This: https://doi.org/10.1021/acsomega.1c03547

Read Online

\begin{abstract}
ACCESS
山ll Metrics \& More

回 Article Recommendations

S1 Supporting Information

ABSTRACT: Merging of electronics with biology, defined as bioelectronics, at the nanoscale holds considerable promise for sensing and modulating cellular behavior. Advancing our understanding of nanobioelectronics will facilitate development and enable applications in biosensing, tissue engineering, and bioelectronic medicine. However, studies investigating the electrical effects when merging wireless conductive nanoelectrodes with biology are lacking. Consequently, a tool is required to develop a greater understanding of merging conductive nanoparticles with cells. Herein, this challenge is addressed by developing an impedimetric method to evaluate bipolar electrode (BPE) systems that could report on electrical input. A theoretical framework is provided, using impedance to determine if conductive nanoparticles can be polarized and used to drive current. It is then demonstrated that $125 \mathrm{~nm}$ of gold nanoparticle (AuNP) bipolar electrodes (BPEs) could be sensed in the presence of cells when incorporated intracellularly at $500 \mu \mathrm{g} / \mathrm{mL}$ using water and phosphate-buffered saline (PBS) as electrolytes. These results highlight how nanoscale BPEs act within biological systems. This research will impact the rational design of using BPE systems in cells for both sensing and actuating applications.
\end{abstract}

\section{INTRODUCTION}

We are entering a new era where bioelectronic tools enable us to harness and modulate cellular electricity. This allows us to control and sense cell behavior for various applications, from biosensing, microbial fuel cells, and to the treatment of disease. ${ }^{1-3}$ One of the remaining challenges to be overcome to further advance this area is to develop new nanobioelectronics capable of interfacing with cells at an equivalent spatial level of cellular electronic components, which can be operated remotely in a noninvasive manner. Also key is the development of new sensing systems capable of measuring cellular bioelectronic inputs and outputs. Moreover, advancing this area will lead to new opportunities that may couple electrical input to mechanical and optical outputs, as recently reviewed, ${ }^{4}$ with obvious sensing and actuation impacts. In doing so, this will shed light on how bioelectricity underpins cell behavior. To date, the rational design of wireless nanobioelectronic systems has been limited due to the lack of label-free analytical techniques to study electrical effects on conductive nanosized objects when combined with biological cells. Therefore, one of the aims of this work was to address this particular scientific challenge and to further explore how electric fields interact with nanoscale bioelectronic systems (Figure 1).

We have recently reported on the development of a nanobioelectronic system that was based on using nanobipolar electrodes (nano-BPEs) in the presence of biological cells for the first time..$^{5}$ This work was based on a "bipolar electrochemical system" (BES), which consists of bipolar electrodes (BPEs) and an aqueous electrolyte solution. ${ }^{6-9}$ Upon the application of an external electric field from feeder electrodes (FEs), the BPE becomes polarized. This means that the BPE has an induced positive and negative pole generating the thermodynamic driving force that can result in the ability to mediate redox reactions (Figure 1A). This ability to control redox reactions in cells has the potential for considerable therapeutic impact in diverse fields (Figure 1B). We demonstrate in this work that gold nanoparticles (AuNPs) act as bipolar electrodes due to the ability to sense external electric fields and then act as reporters via changes in electronic properties, which we sense by impedance spectroscopy (Figure 1C).

The use of BPEs in electrochemistry is widespread. ${ }^{10-16}$ Over the last decade, new interest has revitalized the field with exciting applications at the micro/nanoscale. ${ }^{17-20}$ Bipolar electrochemistry is also a particularly attractive technique in electroanalysis applications due to its ability to wirelessly address multiple electrodes simultaneously. This allows for multiple detections to be carried out at once using a single pair of feeder electrodes. Such analytical applications make up a

Received: July 6, 2021

Accepted: September 17, 2021 


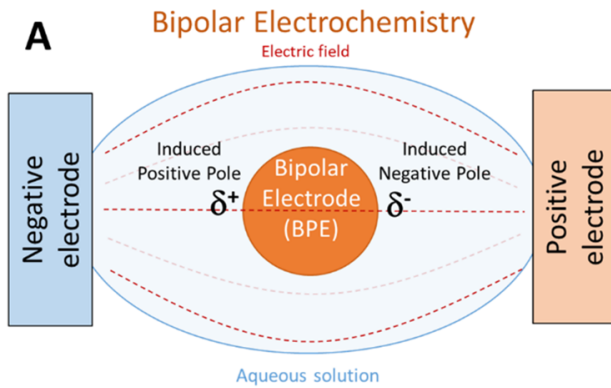

Clectrochemical Impedance Spectroscopy to assess Nano and Intra-cellular BPEs
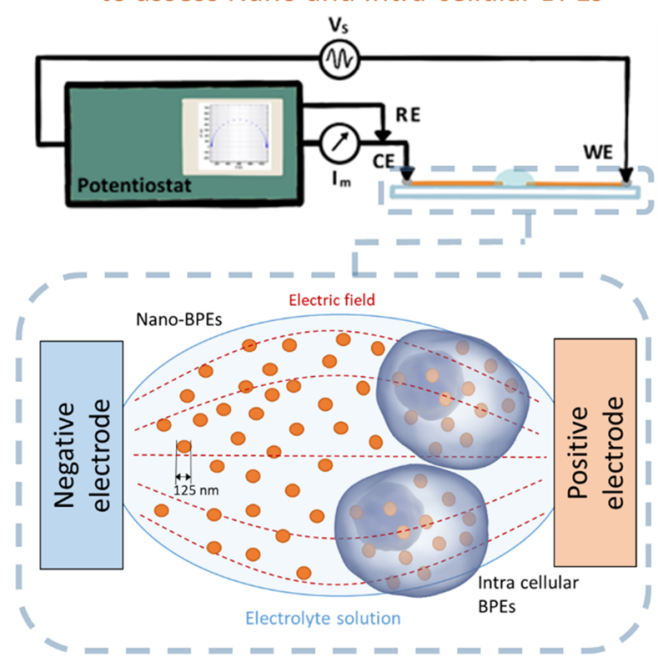

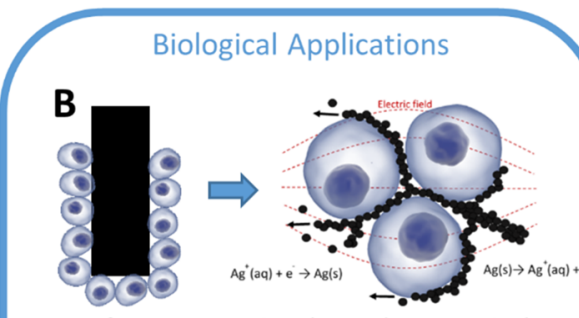

Move from macro wire electrodes to equivalent scale, in-situ grown, bioelectronics (Sanjuan Alberte et al.)

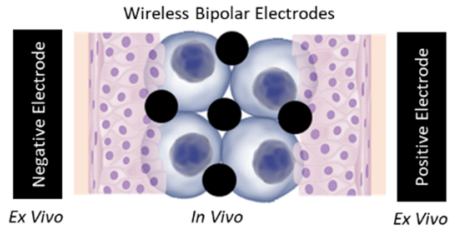

Move from implantable devices and electrodes to less invasive, wirelessly actuated, bipolar electrodes.

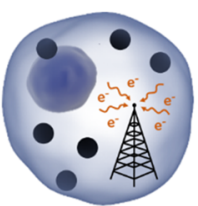

Develop cellular sensing systems, capable of measuring bioelectricity, to shed light on cell behaviours (Sanjuan Alberte et al.)

Figure 1. Interaction of electric fields with nanoscale bioelectronic systems. Schematic showing a bipolar electrochemical cell (A); applications of optimizing bipolar electrochemistry in vivo (B) and the experimental setup used herein to evaluate nano- and nanobioelectronic bipolar systems (C).

large area of the field and have been developed for both chemical and biological analytes. Examples have been recently reviewed. .1,22 $^{210}$

Biological applications of BPEs have been used for the detection, ${ }^{23}$ imaging, ${ }^{24}$ capture, ${ }^{25}$ and lysis ${ }^{26}$ of living cells. Many biological applications focus on the surface of cells or require cell lysis to analyze the intracellular content. One example of probing the intracellular space using bipolar electrochemistry involves piercing individual cells to sense hydrogen peroxide, glucose, or SMase. ${ }^{27}$ However, this does not fully exploit bipolar electrochemistry's wireless nature and high-throughput capabilities. By doing so, we may develop novel noninvasive intracellular bioelectronic systems that could create the new sensing paradigm needed to advance modern medicine and the field of bioelectronics. ${ }^{28}$ This may be possible through the use of nanoparticles as "nano-BPEs".

Nano-BPEs have been largely neglected in open BESs due to the assertation that to polarize nano-BPEs a relatively high voltage was required (in the $\mathrm{kV}$ region). ${ }^{29}$ This has been addressed by confining the BPEs within a nanochannel or pipette..$^{27,30,31}$ However, most recent research has indicated that bipolar electrodes are affected at much lower voltages than expected $(\sim 10 \mathrm{~V}) .^{32}$ These promising results suggest that nano-BPEs may be compatible with biology and therefore open up a wide range of possible applications (Figure 1B). In vivo nanobipolar electrochemistry could be used to grow in situ bioelectronics, ${ }^{5}$ allowing for improved merging with individual cells. As nano-BPEs could be injected and wirelessly actuated, they may also bring new avenues of bioelectronic therapies to areas where implantable electrodes and devices are not possible. In vitro nanobipolar electrochemistry could be used to develop cellular sensing systems, particularly live cell, intracellular sensors that could shed light on the complexities of bioelectricity. This could add insight to the link between cell functions and bioelectricity, having great impacts on our understanding of the cancer phenotype. ${ }^{33}$

Although our previous body of work demonstrated the use of direct current to wirelessly drive current at an intracellular $\mathrm{BPE},{ }^{32}$ we believe that moving toward using alternating current could allow for better prospects of merging with biological systems due to the greater flexibility in tuning potentials by altering frequencies. Hence, the work herein aims to better understand the effect of alternating current on BESs, particularly those with intracellular nano-BPEs.

Other approaches exist to understand BPEs and optimize their use by facilitating carefully designed criteria to allow us to predict current flow through the BPEs. One approach includes equivalent circuit modeling using data from split BPEs or bespoke bipolar cells. ${ }^{34-36}$ Translating these methods to the nanoscale would be extremely difficult due to the need to manufacture electronics to connect to individual nano-BPEs. Another possible method is electrochemically modulated fluorescent probes. ${ }^{37}$ However, as this is an indirect method, it is difficult to produce quantitative information and only BPEs are analyzed, thus neglecting other cell elements (electrolyte/feeder electrodes). This approach also adds the process of fluorescently tagging electrodes and typical fluorescence imaging issues such as photobleaching and 

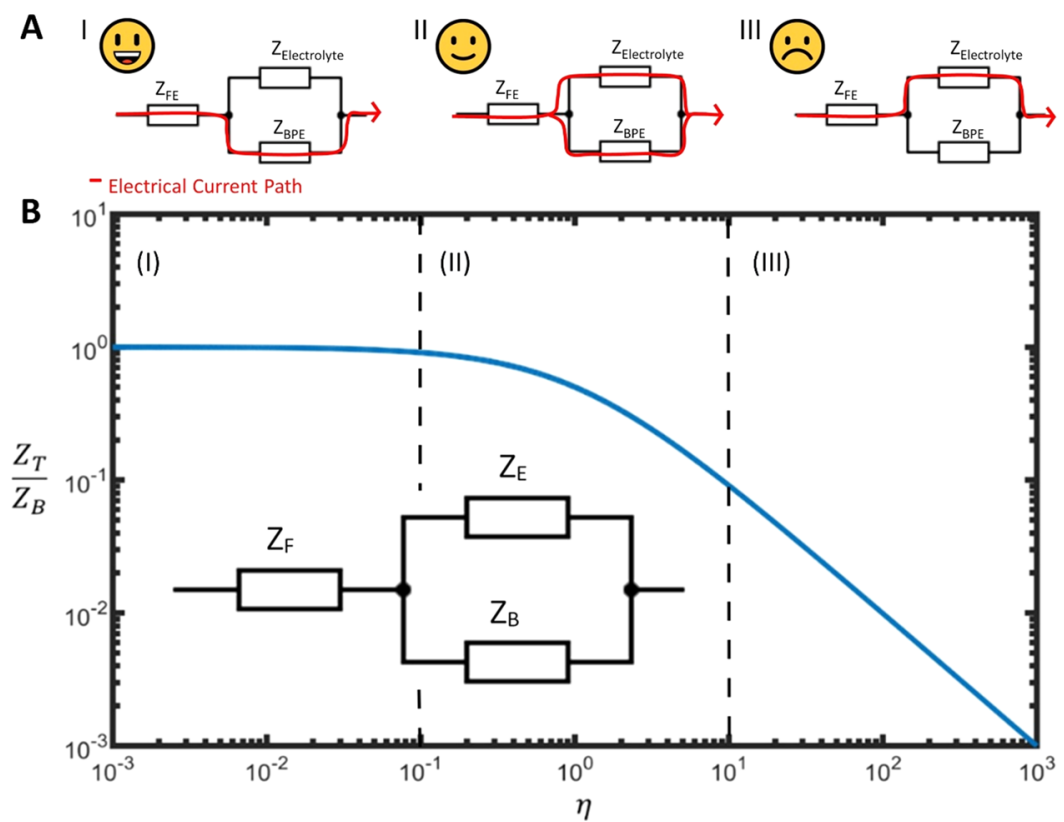

Figure 2. Basis of EIS method to evaluate bipolar electrochemical systems by evaluating the ratio of BPE impedance and electrolyte impedance. Total BES impedance $\left(Z_{\mathrm{T}}\right)$, normalized by BPE impedance $\left(Z_{\mathrm{B}}\right)$, is plotted against efficiency parameter $\eta$ (defined in eq 1$)$ (B). An equivalent circuit can be seen in the inset, with impedance components relating to the feeder electrodes $Z_{\mathrm{F}}$, the electrolyte $Z_{\mathrm{E}}$, and the BPEs $Z_{\mathrm{B}}$. Dotted lines show the three distinct areas (I-III) where current flows mostly through the BPEs, is split between BPE and electrolyte, and mostly flows through the electrolyte (A).

subsequent irreversibility. Hence, an alternative analytical method is required that can be easily applied to nanoscale BESs. We hypothesized that electrochemical impedance spectroscopy (EIS) would be suitable for studying the electrical components of nano-BPE systems as it allows for the assessment of a BES in terms of its electrical circuit behavior, without the need for physical connections to each component in the system.

EIS is a powerful tool capable of deconstructing total impedance into its capacitive, resistive, and inductive contributions $^{38}$ and has been extensively used to study corrosion. $^{39}$ Other applications include material characterization, ${ }^{40,41}$ battery and fuel cell development, ${ }^{42-44}$ and bipolar membrane characterization. ${ }^{45}$ In more recent years, the tool has drawn interest in the life sciences field due to its ability to reveal electrochemical properties such as diffusion coefficients, electron transfer rate constants, adsorption mechanisms, and charge-transfer resistances. ${ }^{46}$ These applications are dominated by EIS-based biosensors, for example, for label-free sensing of DNA $^{47}$ tuberculosis, ${ }^{48}$ possible identification of osteoporosis, ${ }^{49}$ and identification of thyroid cancer using "EIS on a needle" (EON). ${ }^{50,51}$ EIS has also been widely applied to living cells to noninvasively count, identify, or monitor cellular functions; ${ }^{52}$ state-of-the-art instrumentation is now available, allowing the monitoring of cell adhesion, morphology, proliferation, and motion of cells (electrical cell-substrate impedance sensing (ECIS)). ${ }^{53}$ EIS is extremely practical and easy to carry out while also being quickly repeatable. As seen by the wide array of applications, this makes EIS an attractive technique for analytical purposes. Importantly, to the best of our knowledge, it has not been used to study cellular bipolar electrode systems as proposed here and could be a highly valuable technique $^{21}$ (Figure 1).

Herein, we report on a label-free EIS tool for direct modeling of the equivalent circuits of BESs. In our context here, we refer to the nanoparticles as BPEs and use them as capacitive sensors, as the BPEs will align in the field generating a potential difference across the particle. As seen in Figure 1C, this was performed using $125 \mathrm{~nm}$ of AuNP BPEs in electrolytes including water and phosphate-buffered saline (PBS). These nano-BPEs were then combined with biological systems to establish if they could act as reporter BPEs within cells. These results indicate that electrical input parameters can be tuned to drive the electrical polarization of nanoparticles, which could be used to design systems to manipulate or sense cellular biochemistry wirelessly. As well as adding insight into the performance of nano-BPEs, this method also provides a wireless method for probing NPs that could have great sensing applications.

\section{THEORY}

Our first step in designing an analytical tool, which could be used to help us understand how BPEs behave under an electric field, was to model them using a simplified equivalent circuit with three circuit elements. These elements are the impedance of the feeder electrodes $\left(Z_{\mathrm{F}}\right)$, the impedance of the BPEs $\left(Z_{\mathrm{B}}\right)$, and the "bypass impedance" of the electrolyte $\left(Z_{\mathrm{E}}\right)$. Figure 2 (inset) shows the configuration of these components for samples with BPEs without cells.

An impedance-based parameter was then introduced, with the ability to quantitatively describe the efficiency of these BESs in driving current. The efficiency parameter $(\eta)$ is defined as the ratio of the impedance of the BPEs $\left(Z_{\mathrm{B}}\right)$ to the bypass impedance of the electrolyte $\left(Z_{\mathrm{E}}\right)$, as described by eq 1 . This parameter does not include the feeder electrode $\left(Z_{\mathrm{F}}\right)$ as its effect on the overall cell impedance is negligible, exerting an impedance in series with the overall impedance of the bipolar system 


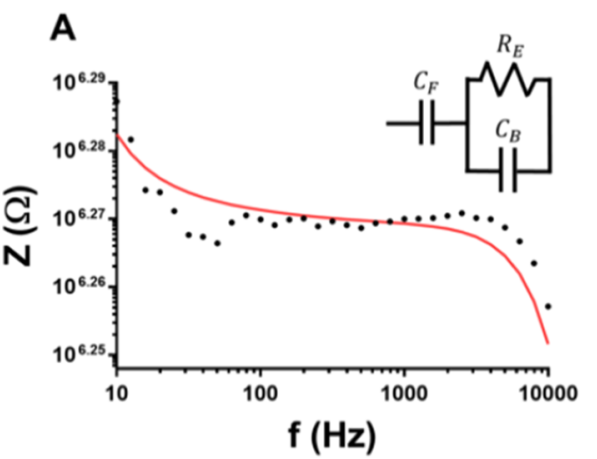

C

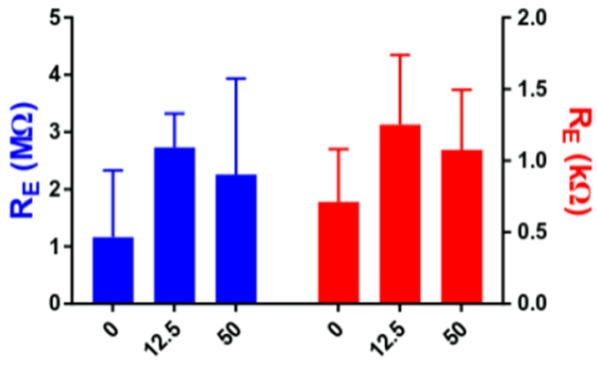

BPE concentration ( $\mu \mathrm{g} / \mathrm{ml})$
B

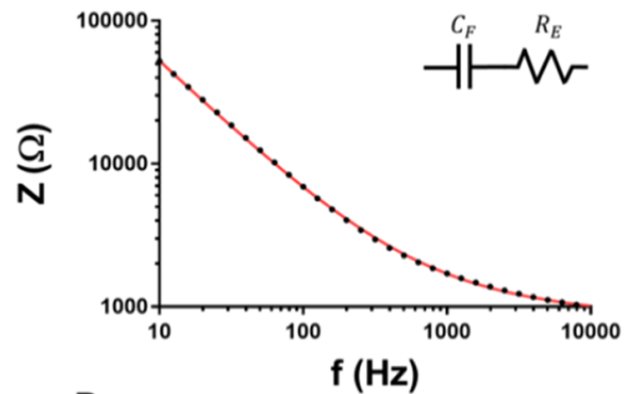

D

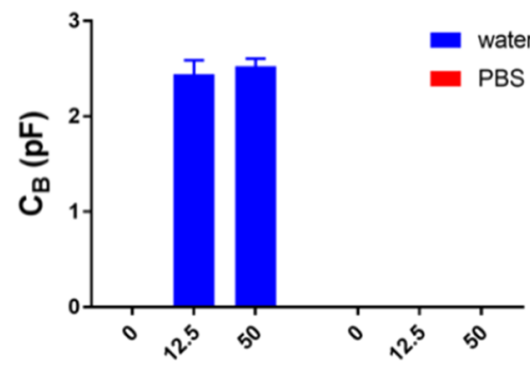

BPE concentration $(\mu \mathrm{g} / \mathrm{ml})$

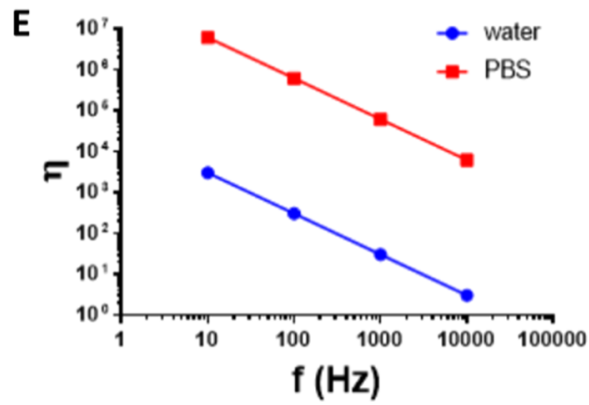

Figure 3. Electrical characterization plots and example circuit fittings of Au feeder electrodes with AuNP bipolar electrodes in water (A) and PBS (B). Circuit diagrams can be seen in the inset. Warburg impedance was also included in series with the feeder electrode to account for diffusion in the system and to allow for better fitting. With $Z_{\mathrm{E}}$ being modeled as a resistor $\left(R_{\mathrm{E}}\right)$ and $Z_{\mathrm{B}}$ modeled as a capacitor $\left(C_{\mathrm{B}}\right)$, panels $(\mathrm{C})$ and $(\mathrm{D})$ show the average magnitudes of $R_{\mathrm{E}}$ and $C_{\mathrm{B}}$, respectively, in samples using water (blue) and PBS (red). $C_{\mathrm{F}}$ averages can be seen in Figure $S 2$. Error bars show the standard error of mean $(n=3)$. Efficiency parameter $(\eta)$ of $125 \mathrm{~nm}$ AuNP BPEs in PBS (blue) and deionized water (red) can be seen in panel (E).

$$
\begin{aligned}
& \eta=\frac{Z_{\mathrm{B}}}{Z_{\mathrm{E}}} \\
& Z_{\mathrm{T}}=\frac{Z_{\mathrm{E}} Z_{\mathrm{B}}}{Z_{\mathrm{E}}+Z_{\mathrm{B}}}
\end{aligned}
$$

To investigate the influence of the efficiency parameter on the total cell impedance, we can model a BES with a fixed $Z_{\mathrm{B}}$ and varying $Z_{\mathrm{E}}$. As seen in Figure 2 , by calculating total impedance $\left(Z_{\mathrm{T}}\right)$, using eq 2 , and normalizing using $Z_{\mathrm{B}}$, we can plot normalized total impedance against the efficiency parameter, giving three distinct regions of $\eta$. The equation can be described in a simple manner: if the electrolyte impedance is significantly lower than the bipolar electrode impedance, then current flows through the electrolyte and vice versa.

In the range where $\eta \geq 10, Z_{\mathrm{T}}$ is dominated by $Z_{\mathrm{E}}$; this case occurs when a highly conductive electrolyte is used (Figure 2A,B: III). Here, current will favor the electrolyte, meaning little to no current would pass through the BPEs, therefore being undesirable in driving electrochemical reactions. As $Z_{\mathrm{E}}$

increases, the electrolyte becomes more resistive, falling within the range of $0.1<\eta<10 ; Z_{\mathrm{T}}$ begins to reflect both $Z_{\mathrm{E}}$ and $Z_{\mathrm{B}}$ (Figure 2A,B: II). This is due to impedance values becoming more similar and hence current tends toward equally splitting between the two components. Finally, when $\eta$ falls below 0.1 , the overall impedance is then limited only by $Z_{\mathrm{B}}$ (Figure $2 \mathrm{~A}, \mathrm{~B}$ : I). This shows that when a highly resistive electrolyte is used, the current would then favor the BPEs, with little to no current passing through the electrolyte. To drive electrochemical reactions at the $\mathrm{BPE}$, and to probe any changes in the BPEs, current is required to pass through the BPEs. Hence, we can conclude that $\eta \leq 10$ must be satisfied when using this analytical method. A similar concept has previously been described, though not using impedance spectroscopy as in this study. ${ }^{29}$ Previous studies required physical connection or labeling of the BPEs to assess electrical behavior. ${ }^{34,36,37}$ Utilizing impedance spectroscopy provides a much simpler, label-free, wireless method that allows the analysis of nanoBPEs for the first time. 


\section{RESULTS AND DISCUSSION}

Before exploring how BESs behave in the presence of cells, we first validated the above theoretical framework by varying electrolytes only. The BES consisted of gold feeder electrodes with spherical AuNP BPEs placed in between. AuNPs are commonly used for in vivo applications; ${ }^{54}$ furthermore, we have recently developed bioelectronic systems using AuNPs in which we observed that they could act as electrical sensors within cells. ${ }^{32}$ They would therefore be suitable for establishing an EIS analytical protocol to study the effects of nanosized BPEs in biological systems. A larger diameter particle is used herein $(125 \mathrm{~nm})$ to allow for higher charge density and compatibility with other techniques, such as assessing plasmonic effects. ${ }^{32}$

Nano-BPE Systems. To test the effect of varying $Z_{\mathrm{E}}$ and hence experimentally validate the model described in Figure 2, samples were tested in high- and low-impedance electrolytes: deionized water and PBS, respectively. A range of concentrations of PBS were also studied to further test the hypothesis that increasing $Z_{\mathrm{E}}$ allows for the increased efficiency of the system $(\eta)$ (Figure S1). It is important to note that this is not to simulate in vivo conditions but to assess how these nanoBESs behave in high- and low-impedance-electrolyte examples.

Subsequently, we then analyzed the resulting frequency impedance plots by least-squared fitting to construct equivalent circuits (Figure 3). Warburg impedance was also included in series with the feeder electrode to account for diffusion in the system and to allow for better fitting (diffusion of ions). However, it was found that these values were not significantly different between repeats and hence are not discussed. All results satisfied Kronig-Kramer's test, showing that the system is linear and stable with time, which is a prerequisite for modeling. Both FEs and BPEs are adequately described by a capacitor that represents the electrochemical double layer in the absence of charge transfer across the interface, and therefore, $Z_{\mathrm{F} / \mathrm{B}}$ are modeled as $C_{\mathrm{F} / \mathrm{B}}$. When deionized water is used as an electrolyte, the impedance of AuNPs is lower in comparison to that of $Z_{\mathrm{E}}$, resulting in current flow through the BPEs. As the current passes through BPEs capacitively, an additional capacitor appears in the equivalent circuit accounting for this effect (Figure 3A). In contrast to deionized water, when PBS is used, most current flows through the electrolyte, and the impedance of BPEs is relatively high and therefore behaves as an open circuit (Figure 3B). Due to the practicality of this method, equivalent circuits are much easier to produce when compared to previous methods for probing BPEs. ${ }^{34-36}$

Frequency vs Impedance plots are shown in Figure 3A,B for deionized water and PBS, respectively, confirm that the BESs do behave differently with varying electrolyte impedance. In water, there are three distinct sections of the impedance spectrum. At low frequencies, the impedance is limited by the capacitive nature of the feeder electrodes. This shows a typical frequency-dependent behavior, described in eq 3, where impedance drops by increasing frequency. The second region is characterized by a constant impedance occurring within the frequency range $100-3000 \mathrm{~Hz}$. This component arises from the resistance of charge flow (i.e., $Z_{\mathrm{E}}$, electrolyte impedance). When this is described in terms of the theoretical framework, previously described in Figure 2, the parameter $\eta \geq 10$ for this region. This means, within this region, the electrolyte impedance is still relatively lower than the BPE impedance and therefore is limiting the overall impedance. As the BPE is also behaving as a capacitor, its impedance is also frequencydependent; hence, the impedance of the BPEs becomes less than the impedance of the electrolyte for the frequency range $\sim 3000-10000 \mathrm{~Hz}$. This means current flows through the BPE when $\eta \leq 10$ is satisfied. It can therefore be concluded that to probe these nano-BPEs using impedance spectroscopy within deionized water, measurements should be performed at frequencies higher than $\sim 3000 \mathrm{~Hz}$ when relatively low voltages are applied. Although the reported frequency band is only suited to the BES described above, the concept can be adapted to probe remotely induced electrochemical reactions. To achieve this, a suitable frequency region should be identified since the electrochemical impedance of the BPE-electrolyte interface changes with redox charge transfer. It is worth noting that there is a possibility of driving current at low frequencies if higher voltages are applied, as we have observed previously. ${ }^{32}$ Within PBS (Figure 3B), we only observe two disparate sections of the curve relating to (i) the capacitive effect of the FEs at lower frequencies and (ii) plateauing to the resistive effect of the electrolyte at high frequencies. As expected, the addition of BPEs does not significantly change the impedance spectra, indicating that we are working within the region of $\eta \geq$ 10 with little to no current flowing to the BPEs. As within deionized water, it is expected that at much higher frequencies the BPEs may then be able to be sensed due to their decreasing impedance

$$
Z=\frac{1}{j \omega c}
$$

where $j=\sqrt{ }-1, \omega$ is the angular frequency or $2 \pi f$, and $c$ is capacitance.

We were then interested in determining how the different BES components behave, which would yield knowledge to better understand and design systems. The average of the equivalent circuit components from three independent repeats is plotted in Figure 3C,D. These plots present the electrolyte resistance $\left(R_{\mathrm{E}}\right)$ and the BPE capacitance $\left(C_{\mathrm{B}}\right)$, respectively. FE behavior is not discussed here as it has low impedance in series with the BES (Figure S2) and does not greatly affect the BPEs. As seen in Figure 3C, the resistance of deionized water is on the order of $M \Omega$, as expected due to the low concentration of ions; values are reasonable as the $\mathrm{dH}_{2} \mathrm{O}$ resistance is rated at $18.2 \mathrm{M} \Omega \mathrm{cm}$ and there is a $1 \mathrm{~mm}$ gap between electrodes. PBS impedance is on the order of $\mathrm{k} \Omega$, also reasonable for this electrolyte concentration. ${ }^{55}$ The results presented show that the electrolyte impedance is not affected by the presence or absence of the BPEs, as no significant difference in resistance is witnessed. This observation further confirms the robustness of the technique in extracting the components of the BES. As anticipated from the theoretical framework in Figure 2, the BPEs are detected in the deionized water, rather than in PBS, as presented in Figure 3D. The BPE component was not included when modeling samples in PBS and with no BPEs due to markedly better fitting being achieved (this can be seen in Figure S3). AuNP BPEs were used in two concentrations to determine if the method was sensitive to such differences: 50 and $12.5 \mu \mathrm{g} / \mathrm{mL}$. No difference was seen between these two concentrations, which we believe to be due to the signal-tonoise ratio. We later use a concentration of $500 \mu \mathrm{g} / \mathrm{mL}$ as we expected that a higher magnitude in concentration would show a change in capacitance values allowing for intracellular experiments. 

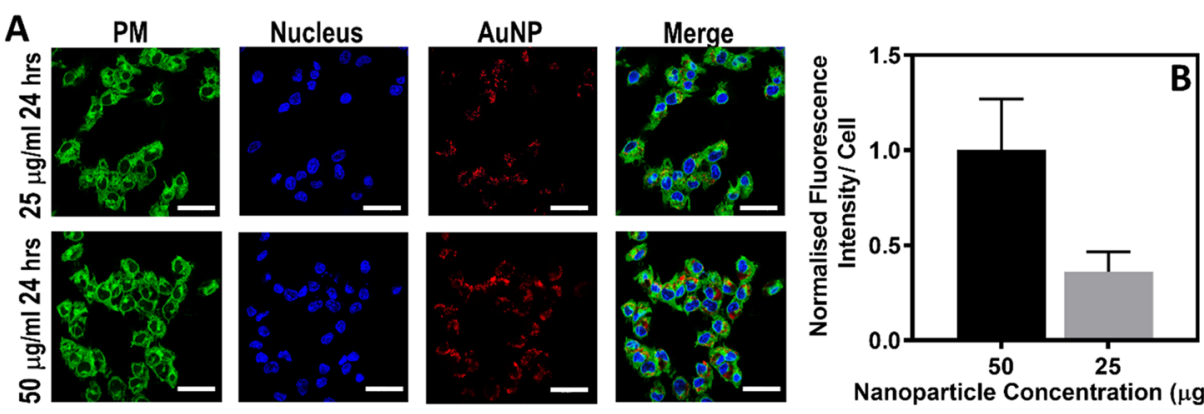

Nanoparticle Concentration $(\mu \mathrm{g} / \mathrm{mL})$
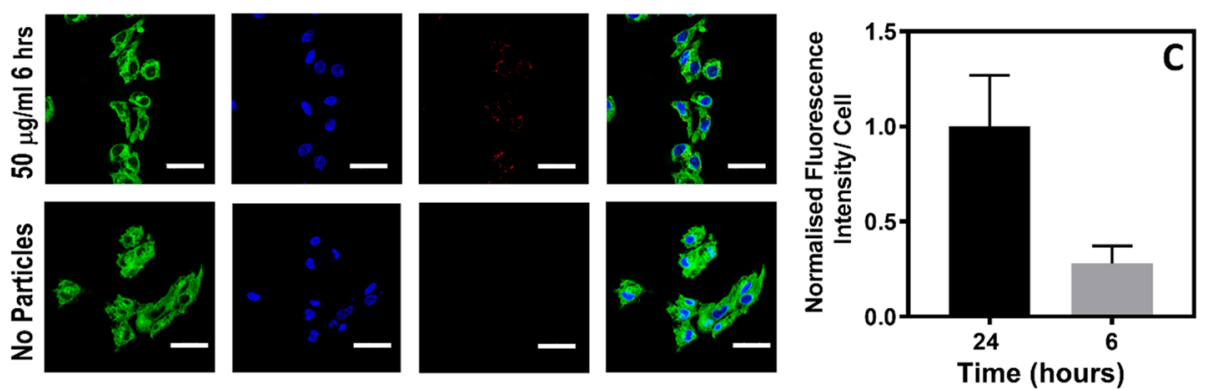

Figure 4. Cellular uptake analysis of AuNP BPEs comparing two concentrations of AuNPs and two incubation times. Confocal microscopy was used to image U251 cells incubated with AuNPs conjugated with Zn porphyrin (A). All scale bars represent $50 \mu$ m. Mean cell fluorescent intensity as a function of cellular uptake and standard deviation (SD) was plotted for at least 20 cells (B, C). All of the values were normalized with fluorescent intensity obtained with a AuNP concentration of $50 \mu \mathrm{g} / \mathrm{mL}$ (B) or a AuNP incubation time of $24 \mathrm{~h}(\mathrm{C}) .50 \mu \mathrm{g} / \mathrm{mL}$ of AuNP concentration was taken forward from panel (B) to perform the incubation time study in panel (C).

Given the capacitance of BPEs calculated from equivalent circuits in Figure 3D $(\sim 2.5 \mathrm{pF})$, we then calculated the efficiency of these BPEs in different electrolyte systems by calculating $\eta$ (using eqs 1 and 3 ). It is important to note that the capacitance of the BPEs would change in PBS and an estimate of this value was not possible due to no current flowing through the BPEs. However, when applying eqs 1 and 3 , even a magnitude difference in capacitance will have little effect on $\eta$; therefore, this will allow a rudimentary comparison between water and PBS. This comparison, seen in Figure 3E, shows that $\eta$ falls below 10 when in water at frequencies higher than $\sim 3000 \mathrm{~Hz}$, again suggesting that we can drive current through these BPEs under these conditions and not when using PBS. As expected, this highlights the need for a highimpedance electrolyte for these AuNP BPEs to be addressed by EIS in sensing applications.

Living Cells with Nano-BPEs. The development of bioelectronic systems that use conductive NPs to sense and actuate cellular behavior would be an extremely important advancement for cancer diagnosis and treatment. ${ }^{56,57}$ Consequently, we used the assay to study how AuNP BPEs behave when placed within U251 malignant brain cancer cells. To determine whether the current would flow through BPEs when cells are present, U251 cells were seeded onto samples without BPEs, as well as with intracellular BPEs. Again, high- and lowimpedance electrolytes of water and PBS, respectively, were used. We have previously shown that the short time frame of these experiments ( $<1 \mathrm{~min})$ using water does not affect cell viability. $^{5}$

To provide intracellular BPEs, cells were incubated with AuNPs, before seeding cells onto FEs. As seen in Figure 4, the cellular uptake of AuNPs was confirmed by confocal microscopy. To achieve this, we covalently conjugated fluorescent zinc(II) 5-(4-aminophenyl)-10,15,20-(tri-4sulfonatophenyl)porphyrin triammonium (zinc porphyrin) to these AuNPs (Figure S4). For comparison purposes, two concentrations of AuNPs were tested along with two incubation times: 50 or $25 \mu \mathrm{g} / \mathrm{mL}$ and 6 or $24 \mathrm{~h}$. Both the higher concentration $(50 \mu \mathrm{g} / \mathrm{mL})$ and the longer time frame (24 h) showed significantly higher AuNPs inside cells, and hence, $50 \mu \mathrm{g} / \mathrm{mL}$ for $24 \mathrm{~h}$ was used for experiments herein. We also performed inductively coupled plasma mass spectroscopy (ICP-MS) for cellular uptake analysis of AuNPs to support the obtained confocal data (Figure S5). Considering both confocal imaging and ICP-MS results, using porphyrin-functionalized and bare AuNPs, respectively, we can be confident that AuNPs are being taken up by the cells. To ensure any effects were not as a result of impedance changes associated with the AuNPs toxicity, toxicity was analyzed using a membrane dye (Hoechst/propidium iodide) and a metabolic activity assay (WST-8). All used concentrations of AuNPs had no significant effect on viability and little effect on metabolism, with metabolic activity not dropping below $80 \%$ (Figure S6). This agrees with others who have shown that high AuNP consternations do not significantly affect cell metabolism. ${ }^{58}$

Despite EIS being applied to many biological applications, to the best of our knowledge, only one previous study exists comparing the impedance of tissues with and without the presence of conductive objects (AuNPs). ${ }^{59}$ Measurements are taken at low frequencies and circuits are not fitted, so the behavior of such AuNPs cannot be quantified. Herein, impedance measurements were taken covering a higherfrequency range up to $10 \times 10^{7} \mathrm{~Hz}$. This fully covers the range of frequencies usually used to study cells using EIS and allowed us to fit circuits to better explain the systems. ${ }^{60}$ Highfrequency impedance has previously been shown to be able to be used to study nonlinear systems, which is likely to be the case when cells are present. ${ }^{61}$ Furthermore, the higher frequencies are believed to be more affected by cell membranes, whereas lower frequencies are more affected by solution channels under and between adjacent cells. ${ }^{60}$ It is also reported that the impedance of the cell membrane drops 
A

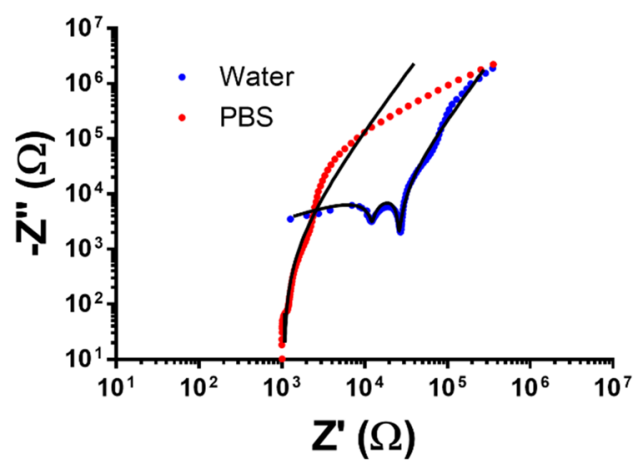

C

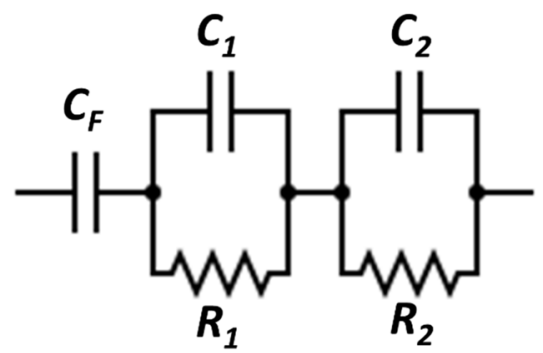

B

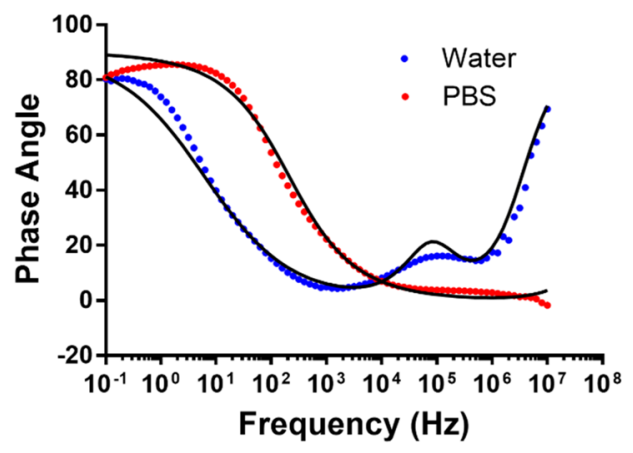

D

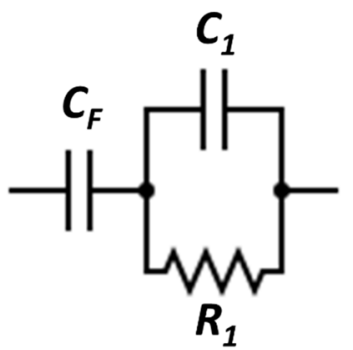

Figure 5. Example data of samples with AuNPs and cells present, using water as an electrolyte. Nyquist plots (A) and phase angle (B) are plotted, with fitting data overlayed in black. Panels (C) and (D) show circuits fitted to water and PBS, respectively.

A

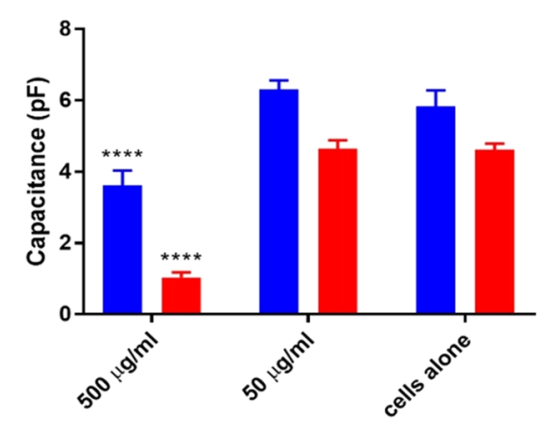

C

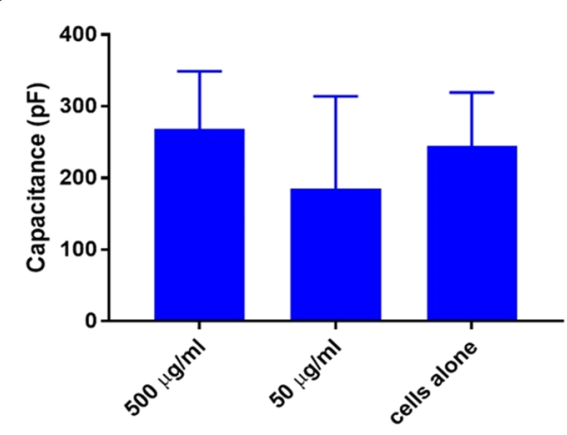

B

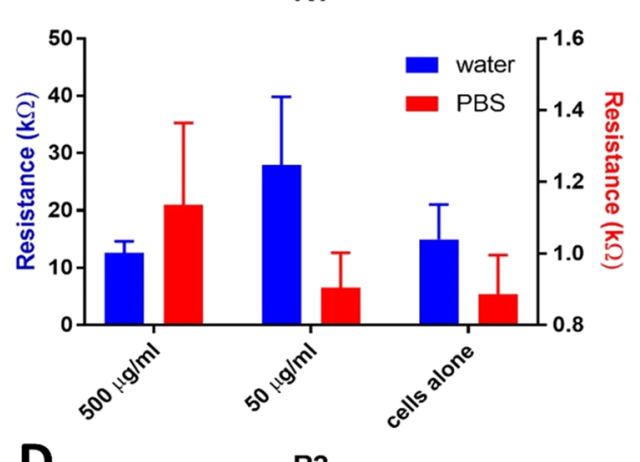

D R2

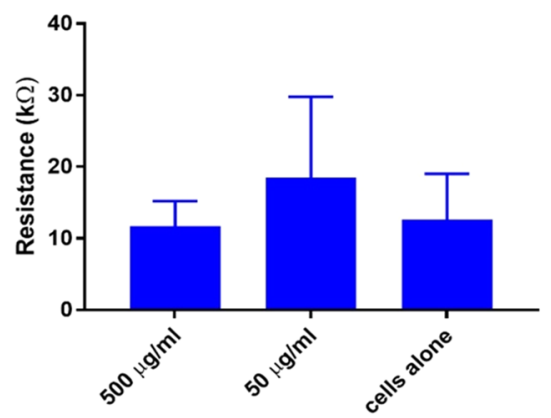

Figure 6. Comparison of fitting values from samples with cells and AuNPs at two concentrations: 50 and $500 \mu \mathrm{g} / \mathrm{mL}$. Capacitance (pF) and resistance $(\mathrm{k} \Omega$ ) values from circuit fittings can be seen using water (blue) and PBS (red) as electrolytes. Circuits used can be seen in Figure 5, with values for circuit elements $C_{1}(\mathrm{~A}), R_{1}(\mathrm{~B}), C_{2}(\mathrm{C})$, and $R_{2}(\mathrm{D})$ plotted. Feeder electrode capacitance values can be found in Figure $S 8$. Error bars show the standard error of the mean $(n=3)$. Significant differences $(*)$ were assessed using a two-way analysis of variance (ANOVA) and Tukey's multiple comparison test.

significantly at megahertz frequencies and it effectively acts as a short circuit. ${ }^{62}$ Therefore, there is a better scope to probe intracellular BPEs at these higher frequencies. Moreover, unlike raw spectra, resistance and capacitance values from fitting are normally distributed, passing a Shapiro-Wilk normality test $(p>0.01)$, allowing us to discern significant differences more easily. The higher concentration was chosen to be studied at high frequencies $(50 \mu \mathrm{g} / \mathrm{mL})$, as well as a 10 - 
fold higher concentration, to maximize the chance of sensing intracellular BPEs $(500 \mu \mathrm{g} / \mathrm{mL})$.

As performed with samples without cells, circuit fittings were carried out to better understand how the systems are behaving. These were approximated fits representing the components of the plots that were indicative of the cells and nanoparticles. Upon analysis of the samples in water, we elucidate that we have two-time constants, represented by the two semicircles in the Nyquist plots, and an increase in the phase angle at higher frequencies (Figure 5A,B). These phenomena are not present in the PBS data, and hence a different circuit was required for the different electrolyte samples. However, both setups are sensitive to the presence of the BPEs inside cells, as demonstrated in Figure 6A. As seen in Figure 5C,D, these circuits included FE capacitance $\left(C_{\mathrm{F}}\right)$, in series with an RC circuit with electrolyte resistance $R_{1}$, and capacitance we believe to be linked to the cells $C_{1}$. Water samples included a second RC circuit with a second resistance and capacitance $\left(R_{2}\right.$ and $C_{2}$ ).

The above simple circuits were fitted to the experimental data, and the circuit components are presented in Figure 6. Sample fitting can be seen in Figure S7. As expected, $C_{F}$ values were not significantly different across all samples and hence will not be discussed (values can be seen in Figure S8). $R_{1}$ values were also not significantly different within the electrolytes, suggesting that the main contributor to this is the resistance of the electrolyte used. We do, however, observe a significant difference in $C_{1}$ values when comparing the highest concentration of AuNPs to the case of the lowest concentration or absence of AuNPs (Figure 6A). Due to this, we can conclude that the capacitance $C_{1}$ is associated with the intracellular ionic differences and therefore the reported analytical method is sensitive to the presence of AuNPs in the intracellular space. This is supported by the evidence we provided that AuNPs are inside the cells, which was demonstrated using ICP-MS and fluorescent studies. If the capacitor was linked to the cellular component alone, then the AuNP concentration would have no effect; therefore, this importantly indicates that we can sense AuNPs inside the cells in both water and PBS. These findings show that measurements can be performed in PBS, which will provide a better mimic of physiological conditions and allow for longer-term studies. We predict that the lower capacitance at higher AuNP concentrations is due to AuNPs agglomerating inside cells. This agglomeration would provide BPEs with a smaller surface area and therefore lower capacitance.

This is the first evidence that AuNPs can act as BPEs to sense electrical input, as previously suggested by us. ${ }^{21}$ This exciting finding suggests that the proposed impedimetric characterization can be used to detect cell-NP interfacing and probe the flow of therapeutic current through these BPEs. This could provide a well-needed new sensing paradigm, whereby functionalized NPs can be used to probe intracellular bioelectricity.

Considering water samples only, we witness a second RC circuit. Again, $R_{2}$ is similar in magnitude to the electrolyte resistance and so this is likely to be the main contributor. As for $C_{2}$, we believe this to be linked to the cells themselves acting as BPEs due to the presence of the AuNPs having no effect on the capacitance.

These results show that within these conditions, nano-BPEs can be used as electrical sensors when in the intracellular space. Coupled with the ease of functionalization on NPs, this could have great intracellular sensing application. Previous BPEbased biosensors often require cell lysis with analytes and then placed onto $\mathrm{BPEs}^{63}$ or the piercing of individual cells with a nanopipette, ${ }^{27}$ whereas the use of EIS as shown herein would allow for noninvasive live monitoring of intracellular BPEs for the first time. Further optimization could allow the generation of useful currents at the BPEs for actuation applications. This could then allow for the use of nano-BPEs in targeting or enhancing electric fields, growing in vivo electronics, and actuating cellular functions (Figure 1B). This could therefore provide an exciting new sensing and actuation paradigm that is needed to progress the field of bioelectronics. ${ }^{28}$

\section{CONCLUSIONS}

This work provides advancing knowledge on the application of nanoscale BPE systems in cells, which until now was lacking. As applied herein, EIS allows a simple way to evaluate a BPE system and model its equivalent circuit. Such a method is both simpler than existing methods, ${ }^{34-37}$ as well as being possible with nano-BPEs for the first time.

Here, we propose an impedimetric method of evaluating the efficiency of a BPE and electrolyte combination. By modeling the total impedance in relation to $\mathrm{BPE}$ and electrolyte impedance, we propose the efficiency parameter $\eta$. We then validate this model experimentally using AuNP BPEs and highand low-impedance electrolytes: deionized water and PBS, respectively. This confirms that within these conditions, these BPEs can be probed in water at high frequencies but not in PBS.

Further to this, we introduced cells to the system to aid in determining if AuNP BPEs can be used as bioelectronics for sensing electrical input. High-frequency measurements were taken to allow for circuit fittings. Within this system, intracellular BPEs at a concentration of $500 \mu \mathrm{g} / \mathrm{mL}$ show a significant difference from cells alone in both water and PBS. This suggests that current is being driven through them. This is a key finding suggesting that nano-BPEs could be used as electrical sensors within the intracellular space and also highlights the efficacy of EIS as a noninvasive technique for probing nano-BPEs, both with and without biology.

\section{EXPERIMENTAL SECTION}

Electrode Manufacture. All electrodes were manufactured in-house. Feeder electrodes were fabricated using a Peltier-cooled dual-target sputter coater (Emitech k575x) and three-dimensional (3D) printed poly(lactic acid) (PLA) masks (printed using an Ultimaker). As seen in Figure 7, electrodes were approximately $2 \mathrm{~mm}$ in width with a $1 \mathrm{~mm}$ gap between them. Sputter coating consisted of first coating with $20 \mathrm{~nm}$ of indium tin oxide (ITO) (at $30 \mathrm{~mA}$ ), as an adhesion layer, before coating with $100 \mathrm{~nm}$ (at $120 \mathrm{~mA}$ ) of gold. Accurate spherical citrate-stabilized gold nanoparticles (AuNPs) were purchased from Nano Partz with a diameter of $125 \mathrm{~nm}$. Nanobipolar electrodes were prepared using an electrostatic coating of AuNPs in between the feeder electrodes at two concentrations: 50 and $12.5 \mu \mathrm{g} / \mathrm{mL}$. First, $20 \mu \mathrm{L}$ of potassium hydroxide solution ( $1 \mathrm{M} \mathrm{KOH}$ in $60 \%$ ethanol) was pipetted between feeder electrodes and left for $1 \mathrm{~h}$. Following this step, two synthetic polymers with opposite charges, poly(allylamine hydrochloride) (PAH) and poly(sodium 4-styrenesulfonate) (PSS), were deposited for $30 \mathrm{~min}$ each. Polymer solutions were both $0.1 \mathrm{M}$ in $\mathrm{dH}_{2} \mathrm{O}$. Finally, $20 \mu \mathrm{L}$ of AuNPs was left to drop- 


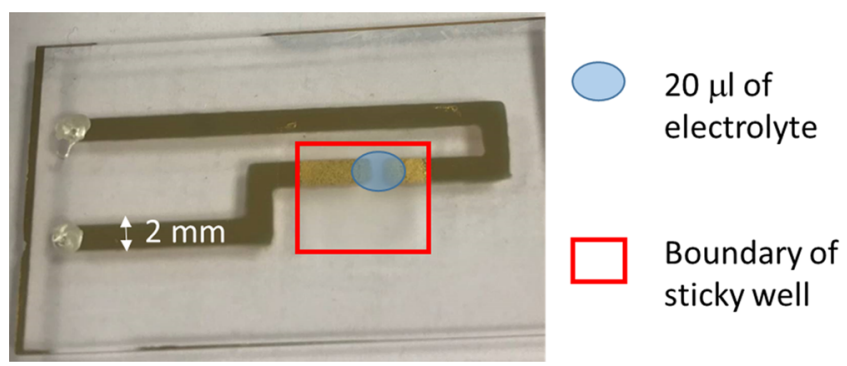

Figure 7. Au feeder electrode setup. Feeder electrodes were approximately $2 \mathrm{~mm}$ in width, with a $1 \mathrm{~mm}$ gap between them. Twenty microliters of electrolyte was used to bridge the gap between electrodes; the approximate boundary of this droplet can be seen in blue. Samples with cells were produced using Ibidi sticky slides, creating an approximate area of a cell coverage of $1 \mathrm{~cm}^{2}$; this can be seen in red. Twenty microliters of electrolyte was also added; however, this spread across the entire cell surface.

coat overnight. Between all steps, and following AuNP modification, the electrodes were washed with $\mathrm{dH}_{2} \mathrm{O}$ and dried with nitrogen.

EIS Measurements. All impedance measurements were carried out using an Autolab potentiostat (Metrohm) with an applied AC amplitude of $0.01 \mathrm{~V}_{\mathrm{RMS}}$ in potentiostatic mode. A two-electrode system was used, with a working electrode and reference/counter electrodes combined. Impedance was measured between 10 and $10000 \mathrm{~Hz}$, with a current limit set at $1 \mathrm{~mA}$. For high-frequency measurements, a highfrequency module, attached to the potentiostat, was used (ECI10M). Ag conductive paint (RS components) was used to create larger contact areas to attach samples to the potentiostat via crocodile clips. A single droplet of $20 \mu \mathrm{L}$ of electrolyte was used, as this was enough to just bridge the gap between feeder electrodes.

Surface Functionalization of Gold Nanoparticles. Zinc(II) 5-(4-aminophenyl)-10,15,20-(tri-4-sulfonatophenyl)porphyrin triammonium (zinc porphyrin) was covalently attached on gold nanoparticles (AuNPs) using 1-ethyl-3-(3dimethylaminopropyl)carbodiimide (EDC)/N-hydroxysuccinimide (NHS) coupling chemistry. In the first step, AuNPs were functionalized with 11-mercaptoundecanoic acid (MUA) to introduce carboxylic groups. Briefly, $1 \mathrm{~mL}$ of MUA (100 $\mu \mathrm{M})$ in 1:9 ethanol/water was added to $5 \mathrm{~mL}$ of citrate-capped AuNPs $(100 \mu \mathrm{g} / \mathrm{mL})$. The solution was stirred overnight at room temperature (RT) under a $\mathrm{N}_{2}$ atmosphere. Later, the AuNPs were washed three times with water and centrifuged at $2500 \mathrm{rpm}$ for $20 \mathrm{~min}$ to obtain MUA-capped AuNPs. In the second step, freshly prepared $1 \mathrm{~mL}$ of EDC $(100 \mathrm{mM})$ and 1 $\mathrm{mL}$ of NHS (200 $\mathrm{mM})$ were added to the MUA-capped AuNPs to activate the carboxyl groups. The reaction was allowed to continue for $4 \mathrm{~h}$ under $\mathrm{N}_{2}$. Then, $1 \mathrm{~mL}$ of zinc porphyrin $(100 \mu \mathrm{M})$ was added to this mixture, and the solution was stirred at room temperature for $24 \mathrm{~h}$ under dark. Finally, the AuNPs were washed three to four times with water and centrifuged at $2500 \mathrm{rpm}$ for $20 \mathrm{~min}$ to obtain AuNPs conjugated with zinc porphyrin.

Cell Culture Studies. Human glioblastoma cell line (U251s obtained from ATCC) was cultured and expanded under standard cell culture conditions, antibiotic-free, using culture medium (Dulbecco's modified Eagle's medium (DMEM); Thermo Fisher Scientific) supplemented with $10 \%$ fetal bovine serum (FBS; Thermo Fisher Scientific) and
1\% L-glutamine (Thermo Fisher Scientific). The cells were passaged using trypsin. Cells were cultured onto microelectrode samples using Ibidi sticky slides at 500000 cells and left for 4-6 h to attach before running EIS measurements. For EIS measurements, sticky slides were removed; all traces of media were first washed away by submerging samples in deionized water, and then $20 \mu \mathrm{L}$ of electrolyte was added. During all experiments, deionized water $(18.2 \mathrm{M} \Omega \mathrm{cm})$ and sterile PBS (0.01 M, Thermo Fischer Scientific) were used.

For cellular uptake analysis, $1.5 \times 10^{4} \mathrm{U} 251$ cells were seeded on a $13 \mathrm{~mm}$ glass viewing area of a $35 \mathrm{~mm}$ glass-bottom dish (Thermo Fisher Scientific) and incubated at $37{ }^{\circ} \mathrm{C}$ for 24 h. After $24 \mathrm{~h}$, the culture medium was replaced with a fresh medium containing 50 or $25 \mu \mathrm{g} / \mathrm{mL}$ AuNPs (functionalized with zinc porphyrin) for 6 or $24 \mathrm{~h}$. After each incubation period, the media containing the AuNPs was removed and cells were washed thrice with PBS. The plasma membrane of the cells was stained using CellMask Green Plasma Membrane Stain (Thermo Fisher Scientific) for $10 \mathrm{~min}$. Later, the cells were fixed with $4 \%$ formaldehyde for $5 \mathrm{~min}$ and subsequently washed three times with PBS. Afterward, cells were treated with 4',6-diamidino-2-phenylindole (DAPI) for $5 \mathrm{~min}$ at RT under dark and washed again thrice with PBS. Finally, the cells were immersed in PBS and imaged using a Zeiss Elyra confocal microscope. Cellular uptake of AuNPs functionalized with zinc porphyrin was quantified by measuring the fluorescent intensity of at least 20 different cells using ImageJ. The fluorescent intensity values were normalized to the total number of cells per field and expressed as corrected total cell fluorescence (CTCF) obtained by applying eq 4

$$
\begin{aligned}
\mathrm{CTCF}= & \text { integrated density }-(\text { area of selected cell } \\
& \times \text { mean fluorescence of background signal })
\end{aligned}
$$

\section{ASSOCIATED CONTENT}

\section{SI Supporting Information}

The Supporting Information is available free of charge at https://pubs.acs.org/doi/10.1021/acsomega.1c03547.

Nyquist plots obtained at varying PBS concentrations were tested in systems with (B) and without (A) AuNP bipolar electrodes; impedance vs frequency plots and fittings of bipolar systems with AuNP BPEs in PBS; histogram of the average magnitudes of feeder electrode capacitance in water (blue) and PBS (red) from circuit fittings used in Figure 3; fluorescent emission spectrum of AuNPs functionalized with zinc porphyrin monitored upon excitation with a wavelength of $422 \mathrm{~nm}$; ICP-MS data to indicate gold nanoparticle uptake inside of the cells at varying concentrations; data detailing the metabolic activity and cytotoxicity data of cells exposed to gold nanoparticles; data and sample fittings of raw data of systems with living cells in water (A) and PBS (B): such fitting was used to determine the resistance and capacitance values seen in Figure 6; and measured capacitance values of the feeder electrodes (PDF)

\section{AUTHOR INFORMATION}

\section{Corresponding Authors}

Sidahmed Abayzeed - Optics and Photonics Research Group, Faculty of Engineering, University of Nottingham, 
Nottingham NG7 2RD, U.K.;

Email: Sidahmed.Abayzeed2@nottingham.ac.uk

Frankie J. Rawson - Regenerative Medicine and Cellular

Therapies, Biodiscovery Institute, School of Pharmacy,

University of Nottingham, Nottingham NG7 2RD, U.K.;

(1) orcid.org/0000-0002-4872-8928;

Email: Frankie.Rawson@nottingham.ac.uk

\section{Authors}

Andie J. Robinson - Regenerative Medicine and Cellular

Therapies, Biodiscovery Institute, School of Pharmacy, University of Nottingham, Nottingham NG7 2RD, U.K.

Akhil Jain - Regenerative Medicine and Cellular Therapies, Biodiscovery Institute, School of Pharmacy, University of Nottingham, Nottingham NG7 2RD, U.K.; 이이.org/ 0000-0003-2019-2030

Ruman Rahman - Children's Brain Tumour Research Centre (CBTRC), Biodiscovery Institute, School of Medicine,

University of Nottingham, Nottingham NG7 2RD, U.K.; (1) orcid.org/0000-0002-6541-9983

Richard J. M. Hague - Centre for Additive Manufacturing, Faculty of Engineering, University of Nottingham, Nottingham NG8 1BB, U.K.

Complete contact information is available at: https://pubs.acs.org/10.1021/acsomega.1c03547

\section{Notes}

The authors declare no competing financial interest.

\section{ACKNOWLEDGMENTS}

This work was supported by the Engineering and Physical Sciences Research Council [grant numbers EP/L015072/1 and EP/R004072/1]. The authors would like to thank Dominic Mosses who performed some initial groundwork leading on to this study and Drs. Scott Young and Saul Vazquez-Reina for their assistance with ICP-MS analysis.

\section{REFERENCES}

(1) Mehrotra, P. Biosensors and their applications - A review. J. Oral Biol. Craniofacial Res. 2016, 6, 153-159.

(2) Rabaey, K.; Verstraete, W. Microbial fuel cells: Novel biotechnology for energy generation. Trends Biotechnol. 2005, 23, 291-298.

(3) Olofsson, P. S.; Tracey, K. J. Bioelectronic medicine: technology targeting molecular mechanisms for therapy. J. Intern. Med. 2017, 282, $3-4$.

(4) Midolo, L.; Schliesser, A.; Fiore, A. Nano-opto-electromechanical systems. Nat. Nanotechnol. 2018, 13, 11-18.

(5) Sanjuan-Alberte, P.; Saleh, E.; Shaw, A. J.; Lacalendola, N.; Willmott, G.; Vaithilingam, J.; Alexander, M. R.; Hague, R. J. M.; Rawson, F. J. Remotely Controlled in Situ Growth of Silver Microwires Forming Bioelectronic Interfaces. ACS Appl. Mater. Interfaces 2019, 11, 8928-8936.

(6) Fosdick, S. E.; Knust, K. N.; Scida, K.; Crooks, R. M. Bipolar electrochemistry. Angew. Chem., Int. Ed. 2013, 52, 10438-10456.

(7) Karimian, N.; Hashemi, P.; Afkhami, A.; Bagheri, H. The principles of bipolar electrochemistry and its electroanalysis applications. Curr. Opin. Electrochem. 2019, 17, 30-37.

(8) Koefoed, L.; Pedersen, S. U.; Daasbjerg, K. Bipolar Electrochemistry - A wireles approach for electrode reactions. Curr. Opin. Electrochem. 2017, 13-17.

(9) Loget, G.; Zigah, D.; Bouffier, L.; Sojic, N.; Kuhn, A. Bipolar Electrochemistry: From Materials Science to Motion and Beyond. Acc. Chem. Res. 2013, 46, 2513-2523.
(10) Backhurst, J. R.; Coulson, J. M.; Goodridge, F.; Plimley, R. E.; Fleischmann, M. A Preliminary Investigation of Fluidized Bed Electrodes. J. Electrochem. Soc. 1969, 116, 1600-1607.

(11) Cervera-March, S.; Smotkin, E. S.; Bard, A. J.; Campion, A.; Fox, M. A.; Mallouk, T.; Webber, S. E.; White, J. M. Modeling of Bipolar Semiconductor Photoelectrode Arrays for Electrolytic Processes. J. Electrochem. Soc. 1988, 135, 567.

(12) Ellis, K. G.; Jansson, R. E. W. W. Further studies on the epoxidation of propylene in a bipolar trickle bed. J. Appl. Electrochem. 1981, 11, 531-535.

(13) Lee, J. K.; Shemilt, L. W.; Chun, H. S. Studies of bipolarity in fluidized bed electrodes. J. Appl. Electrochem. 1989, 19, 877-881.

(14) Manji, A.; Oloman, C. W. Electrosynthesis of propylene oxide in a bipolar trickle-bed reactor. J. Appl. Electrochem. 1987, 17, 532544.

(15) Smotkin, E.; Bard, A. J.; Campion, A.; Fox, M. A.; Mallouk, T.; Webber, S. E.; White, J. Bipolar titanium dioxide/platinum semiconductor photoelectrodes and multielectrode arrays for unassisted photolytic water splitting. J. Phys. Chem. A 1986, 90, 4604-4607.

(16) Wiesener, K.; Ohms, D.; Benczúr-Ürmössy, G.; Berthold, M.; Haschka, F. High power metal hydride bipolar battery. J. Power Sources 1999, 84, 248-258.

(17) Bradley, J. C.; Chen, H. M.; Crawrford, J.; Eckert, J.; Ernazarova, K.; Kurzeja, T.; Lin, M.; McGee, M.; Nadler, W.; Stephens, S. G. Creating electrical contacts between metal particles using directed electrochemical growth. Nature 1997, 389, 268-271.

(18) Loget, G.; Roche, J.; Kuhn, A. True bulk synthesis of Janus objects by bipolar electrochemistry. Adv. Mater. 2012, 24, 51115116.

(19) Loget, G.; Kuhn, A. Electric field-induced chemical locomotion of conducting objects. Nat. Commun. 2011, 2, No. 535.

(20) Perro, A.; Reculusa, S.; Ravaine, S.; Bourgeat-Lami, E.; Duguet, E. Design and synthesis of Janus micro-and nanoparticles. J. Mater. Chem. 2005, 15, 3745-3760.

(21) Rahn, K. L.; Anand, R. K. Recent Advancements in Bipolar Electrochemical Methods of Analysis. Anal. Chem. 2021, 93, 103123.

(22) Bouffier, L.; Zigah, D.; Sojic, N.; Kuhn, A. Bipolar (Bio)electroanalysis. Annu. Rev. Anal. Chem. 2021, 14, 65-86.

(23) Zhang, H.-R.; Wang, Y.-Z.; Zhao, W.; Xu, J.-J.; Chen, H.-Y. Visual Color-Switch Electrochemiluminescence Biosensing of Cancer Cell Based on Multichannel Bipolar Electrode Chip. Anal. Chem. 2016, 88, 2884-2890.

(24) Cao, J.-T.; Wang, Y.-L.; Zhang, J.-J.; Dong, Y.-X.; Liu, F.-R.; Ren, S.-W.; Liu, Y.-M. Immuno-Electrochemiluminescent Imaging of a Single Cell Based on Functional Nanoprobes of Heterogeneous Ru(bpy)32+@SiO2/Au Nanoparticles. Anal. Chem. 2018, 90, 10334-10339.

(25) Li, M.; Anand, R. K. High-Throughput Selective Capture of Single Circulating Tumor Cells by Dielectrophoresis at a Wireless Electrode Array. J. Am. Chem. Soc. 2017, 139, 8950-8959.

(26) Li, M.; Anand, R. K. Integration of marker-free selection of single cells at a wireless electrode array with parallel fluidic isolation and electrical lysis. Chem. Sci. 2019, 10, 1506-1513.

(27) Wang, Y.; Jin, R.; Sojic, N.; Jiang, D.; Chen, H.-Y. Intracellular Wireless Analysis of Single Cells by Bipolar Electrochemiluminescence Confined in a Nanopipette. Angew. Chem., Int. Ed. 2020, 59, 10416-10420.

(28) Rolandi, M.; Noy, A.; Inal, S.; Rivnay, J. Advances in bioelectronics: Materials, devices, and translational applications. APL Mater. 2021, 9, No. 070402.

(29) Loget, G.; Kuhn, A. Shaping and exploring the micro-and nanoworld using bipolar electrochemistry. Anal. Bioanal. Chem. 2011, 400, 1691-1704.

(30) Scida, K.; Eden, A.; Arroyo-Curra, Y.; MacKenzie, S.; Satik, Y.; Meinhart, C. D.; Eijkel, J. C. T.; Pennathur, S. Fluorescence-Based Observation of Transient Electrochemical and Electrokinetic Effects at Nanoconfined Bipolar Electrodes. ACS Appl. Mater. Interfaces 2019, 11, 13777-13786. 
(31) Han, C.; Hao, R.; Fan, Y.; Edwards, M. A.; Gao, H.; Zhang, B. Observing Transient Bipolar Electrochemical Coupling on Single Nanoparticles Translocating through a Nanopore. Langmuir 2019, 35, $7180-7190$.

(32) Sanjuan-Alberte, P.; Jain, A.; Shaw, A. J.; Abayzeed, S. A.; Domínguez, R. F.; Alea-Reyes, M. E.; Clark, M.; Alexander, M. R.; Hague, R. J.; Pérez-García, L.; Rawson, F. J. Wireless Nanobioelectronics for Electrical Intracellular Sensing. ACS Appl. Nano Mater. 2019, 6397.

(33) Robinson, A. J.; Jain, A.; Sherman, H. G.; Hague, R. J. M.; Rahman, R.; Sanjuan-Alberte, P.; Rawson, F. J. Toward Hijacking Bioelectricity in Cancer to Develop New Bioelectronic Medicine. Adv. Ther. 2021, 4, No. 2000248.

(34) Kusakabe, K.; Morooka, S.; Kato, Y. Current paths and electrolysis efficiency in bipolar packed-bed electrodes. J. Chem. Eng. Jpn. 1982, 15, 45-50.

(35) Kusakabe, K.; Morooka, S.; Kato, Y. Equivalent resistances for current pathways in a bipolar packed-bed electrode cell. J. Chem. Eng. Jpn. 1986, 19, 43-47.

(36) Sudoh, M.; Kodera, T.; Hino, H.; Shimamura, H. Oxidative Degradation Rate of Phenol in An Undivided Bipolar Electrolyzer. J. Chem. Eng. Jpn. 1988, 21, 536-538.

(37) Scida, K.; Eden, A.; Arroyo-Curra, Y.; MacKenzie, S.; Satik, Y.; Meinhart, C. D.; Eijkel, J. C. T.; Pennathur, S. Fluorescence-Based Observation of Transient Electrochemical and Electrokinetic Effects at Nanoconfined Bipolar Electrodes. ACS Appl. Mater. Interfaces 2019, 11, 13777-13786.

(38) Macdonald, D. D. Reflections on the history of electrochemical impedance spectroscopy. Electrochim. Acta 2006, 51, 1376-1388.

(39) Epelboin, I.; Keddam, M.; Takenouti, H. Use of impedance measurements for the determination of the instant rate of metal corrosion. J. Appl. Electrochem. 1972, 2, 71-79.

(40) Chen, Z.; He, C.; Yu, F.; Wang, Y. Study and Application of Electrochemical Impedance Spectroscopy for Quickly Evaluating the Performance of Coatings and Predicting the Failure Time in the Development of Waterborne Epoxy Micaceous Iron Oxide Coatings. Int. J. Electrochem. Sci. 2017, 12, 2798-2812.

(41) Loveday, D.; Peterspm, P.; Rodgers, B. Evalution of organic coatings with electrochemical impedance spectroscopy part 2: Application of EIS to coatings. J. Coat. Technol. Res. 2004, 1, 88-93.

(42) Gomadam, P. M.; Weidner, J. W. Analysis of electrochemical impedance spectroscopy in proton exchange membrane fuel cells. Int. J. Energy Res. 2005, 29, 1133-1151.

(43) He, Z.; Mansfeld, F. Exploring the use of electrochemical impedance spectroscopy (EIS) in microbial fuel cell studies. Energy Environ. Sci. 2009, 2, 215-219.

(44) Selman, J. R.; Lin, Y. P. Application of ac impedance in fuel cell research and development. Electrochim. Acta 1993, 38, 2063-2073.

(45) Blommaert, M. A.; Vermaas, D. A.; Izelaar, B.; In'T Veen, B.; Smith, W. A. Electrochemical impedance spectroscopy as a performance indicator of water dissociation in bipolar membranes. J. Mater. Chem. A 2019, 7, 19060-19069.

(46) Randviir, E. P.; Banks, C. E. Electrochemical impedance spectroscopy: An overview of bioanalytical applications. Anal. Methods 2013, 5, 1098-1115.

(47) Kafka, J.; Pänke, O.; Abendroth, B.; Lisdat, F. A label-free DNA sensor based on impedance spectroscopy. Electrochim. Acta 2008, 53, $7467-7474$.

(48) Teengam, P.; Siangproh, W.; Tuantranont, A.; Vilaivan, T.; Chailapakul, O.; Henry, C. S. Electrochemical impedance-based DNA sensor using pyrrolidinyl peptide nucleic acids for tuberculosis detection. Anal. Chim. Acta 2018, 1044, 102-109.

(49) Bhardwaj, P.; Rai, D. V.; Garg, M. L.; Mohanty, B. P. Potential of electrical impedance spectroscopy to differentiate between healthy and osteopenic bone. Clin. Biomech. 2018, 57, 81-88.

(50) Yun, J.; Kang, G.; Park, Y.; Kim, H. W.; Cha, J.-J.; Lee, J.-H. Electrochemical impedance spectroscopy with interdigitated electrodes at the end of hypodermic needle for depth profiling of biotissues. Sens. Actuators, B 2016, 237, 984-991.
(51) Yun, J.; Hong, Y.-T.; Hong, K.-H.; Lee, J.-H. Ex vivo identification of thyroid cancer tissue using electrical impedance spectroscopy on a needle. Sens. Actuators, B 2018, 261, 537-544.

(52) Sun, T.; Morgan, H. Single-cell microfluidic Impedance cytometry: A review. Microfluid. Nanofluid. 2010, 8, 423-443.

(53) Lukic, S.; Wegener, J. Impedimetric Monitoring of Cell-Based Assays. eLS; John Wiley \& Sons, 2015; pp 1-8.

(54) Cabuzu, D.; Cirja, A.; Puiu, R.; Grumezescu, A. Biomedical Applications of Gold Nanoparticles. Curr. Top. Med. Chem. 2015, 15, $1605-1613$

(55) Ribeiro, M.; Elghajiii, A.; Fraser, S. P.; Burke, Z. D.; Tosh, D.; Djamgoz, M.; Rocha, P. R. Human Breast Cancer Cells Demonstrate Electrical Excitability. Front. Neurosci. 2020, 14, No. 404.

(56) Sanjuan-Alberte, P.; Alexander, M. R.; Hague, R. J.; Rawson, F. $\mathrm{J}$. Electrochemically stimulating developments in bioelectronic medicine. Bioelectron. Med. 2018, 4, No. 1.

(57) Sanjuan-Alberte, P.; Rawson, F. J. Engineering the spark into bioelectronic medicine. Ther. Delivery 2019, 10, 139-142.

(58) Mioc, M.; Pavel, I. Z.; Ghiulai, R.; Coricovac, D. E.; Farcaş, C.; Mihali, C.-V.; Oprean, C.; Serafim, V.; Popovici, R. A.; Dehelean, C. A.; Shtilman, M. I.; Tsatsakis, A. M.; Şoica, C. The Cytotoxic Effects of Betulin-Conjugated Gold Nanoparticles as Stable Formulations in Normal and Melanoma Cells. Front. Pharmacol. 2018, 9, No. 429.

(59) Ostovari, M.; Riahi Alam, N.; Zabihzadeh, M.; Gharib-Vand, M. M.; Hoseini-Ghahfarokhi, M. The effect of gold nanoparticles on electrical impedance of tissue on low frequency ranges. J. Biomed. Phys. Eng. 2018, 8, 241-250.

(60) Wegener, J.; Keese, C. R.; Giaever, I. Electric cell-substrate impedance sensing (ECIS) as a noninvasive means to monitor the kinetics of cell spreading to artificial surfaces. Exp. Cell Res. 2000, 259, $158-166$.

(61) Hirschorn, B.; Tribollet, B.; Orazem, M. E. On Selection of the Perturbation Amplitude Required to Avoid Nonlinear Effects in Impedance Measurements. Isr. J. Chem. 2008, 48, 133-142.

(62) Hart, F. X.; Palisano, J. R. The Application of Electric Fields in Biology and Medicine. Electric Field; InTech, 2018; pp 161-186.

(63) Masturah binti Fakhruddin, S.; Ino, K.; Inoue, K. Y.; Nashimoto, Y.; Shiku, H. Bipolar Electrode-Based Electrochromic Devices for Analytical Applications - a Review. Electroanalysis 2021, $33,1-16$ 